\title{
Pancreatic mucinous cystic neoplasm in a transgender patient
}

\author{
Deshka Foster', Mohammad F. Shaikh', Elizabeth Gleeson ${ }^{1}$, Blake D. Babcock', Jianping Lin², Robert T. Ownbey², \\ Mark E. Hysell ${ }^{3}$, Daniel Ringold ${ }^{4}$ and Wilbur B. Bowne ${ }^{1 *}$
}

\begin{abstract}
Background: Cystic pancreatic lesions are increasingly more frequent detected clinical entities. Mucinous cystic neoplasm (MCN) is a hormone-related pancreatic tumor (HRTP) with a strong predominance in young and middle-aged females.

Case presentation: Here, we present the case of a 31-year-old surgically transgendered female-to-male patient with a history of alcoholic pancreatitis, on chronic testosterone therapy. He was found to have a pancreatic MCN and underwent distal pancreatectomy and splenectomy.

Conclusion: To our knowledge, this is the first reported case of a transgender patient with a history of hormone replacement therapy (HRT) and pancreatic MCN. We consider possible mechanisms for the pathogenesis to explain this patient's neoplasm.
\end{abstract}

Keywords: Pancreatic MCN, Transgender medicine, Hormone therapy

\section{Background}

Mucinous cystic neoplasm (MCN) is a hormone-related pancreatic tumor (HRTP) with a strong predominance in young and middle-aged females [1]. In 1996, the World Health Organization and Armed Forces Institute of Pathology provided a formal distinction between intraductal papillary mucinous neoplasms (IPMN) and $\mathrm{MCN}$, stressing the importance of the presence of ovarian stroma to diagnose MCN. The validity of this distinction has been confirmed in subsequent studies [2]. However, the criteria for distinguishing $\mathrm{MCN}$ from other mucinous tumors remain debated [2]. These neoplasms have risk for malignancy and require either close radiological follow-up or resection depending on their size and morphological characteristics [3, 4].

The exact mechanism of how sex hormones affect the development of pancreatic tumors is not entirely understood. Pancreatic tissue expresses estrogen receptors [5], and there are contributory reports of $\mathrm{MCN}$ development during pregnancy [6-10] and in post-menopausal women on hormone replacement therapy (HRT) [11]. There is

\footnotetext{
* Correspondence: wilbur.bowne@drexelmed.edu

'Division of Surgical Oncology, Department of Surgery, Drexel University College of Medicine, 254 N. 15th St, MS 413, Philadelphia, PA 19102, USA Full list of author information is available at the end of the article
}

also evidence that pancreatic tissue expresses testosterone receptors [5]. However, reports of pancreatic MCNs in males are limited and of the reports of men with $\mathrm{MCN}$, most have a history of pancreatitis and/or aberrant hormone levels and sexual phenotypic characteristics $[1,12,13]$. The expression of estrogen and progesterone receptors in MCN among some male patients is perplexing [1]. There are also rare reports of pancreatic MCNs in men or post-menopausal women, which do not show the characteristic ovarian-like stroma. Some suggest that such tumors be called "indeterminate mucin-producing cystic neoplasms" so as to maintain the requirement that MCNs are defined by ovarian-like stroma [11].

Here, we present the case of a 31-year-old surgically transgendered female-to-male patient with a history of alcoholic pancreatitis, on chronic testosterone therapy. He was found to have a pancreatic MCN and underwent distal pancreatectomy and splenectomy. To our knowledge, this is the first reported case of a transgender patient on HRT with pancreatic MCN. We provide a case presentation and propose possible mechanisms, based upon our review of the literature, for the pathogenesis of this patient's neoplasm. 


\section{Case presentation}

The patient is a 31-year-old surgically transgendered, female-to-male patient with a history of multiple prior admissions for alcoholic pancreatitis. He was on chronic, long-term testosterone therapy. He presented complaining of pain in the mid-epigastrium, which had worsened over the prior months, with occasional radiation to the back and shoulders and post-prandial worsening of pain. He denied nausea, vomiting, diarrhea, weight change, or constipation.

Additional past medical history was significant for anxiety and attention deficit hyperactivity disorder. His surgical history included laparoscopic cholecystectomy, tonsillectomy, bilateral mastectomy, total bilateral hysterectomy, and bilateral salpingo-oophorectomy. His family history included leukemia, diabetes mellitus, and cholecystitis. His medications included testosterone cypionate, bupropion, escitalopram, hyoscyamine sulfate, omeprazole, and pancrelipase. He was a former smoker with history of polysubstance abuse including chronic alcoholism.

His physical examination was otherwise non-contributory. Serum CA19-9 level was moderately elevated at $72 \mathrm{u} / \mathrm{ml}$ [reference range <34], lipase was elevated at $128 \mathrm{u} / \mathrm{l}$ [ref 7-60], and amylase was within the normal range at $51 \mathrm{u} / \mathrm{l}$ [ref: 20-101]. Serum IgG4 was negative. Computed tomography (CT) scan of the abdomen and pelvis with pancreatic protocol demonstrated a pancreatic body cystic mass (Fig. 1a, b). Endoscopic ultrasound (EUS) confirmed a $30 \times 33 \mathrm{~mm}$ round multi-septated mass in the pancreatic body suggestive of an MCN (Fig. 2). Aspirated cyst fluid analysis revealed an elevated CEA level of $745 \mathrm{ng} / \mathrm{mL}$.

Of note, 6 years prior to presentation at our institution, the patient was being followed with serial cross-sectional imaging, demonstrating a pancreatic body cystic lesion. Importantly, during this interval period of time, the lesion was noted to alternately increase and decrease in size.

Surgical management included distal pancreatectomy and splenectomy. Specimens were sent for pathologic examination. Serial sectioning of the formalin-fixed specimen revealed a $2 \times 1.8 \times 1.2 \mathrm{~cm}$ septated cyst with a tan-pink, smooth, and glistening inner lining, filled with clear, thin fluid. The cyst was located $0.9 \mathrm{~cm}$ from the proximal pancreatic resection margin, $6.7 \mathrm{~cm}$ from the splenic hilum, and $0.1 \mathrm{~cm}$ from the nearest posterior aspect. It abutted but did not involve the main pancreatic duct (Fig. 3a, b). Microscopic examination showed that the cyst was lined by cuboidal or mucin-producing epithelium and surrounded by the ovarian-like stroma, which was immunohistochemically positive for estrogen and progesterone receptors (Fig. 4a-d). These characteristics are consistent with the diagnosis of $\mathrm{MCN}$. The patient's post-operative course was complicated by a peri-
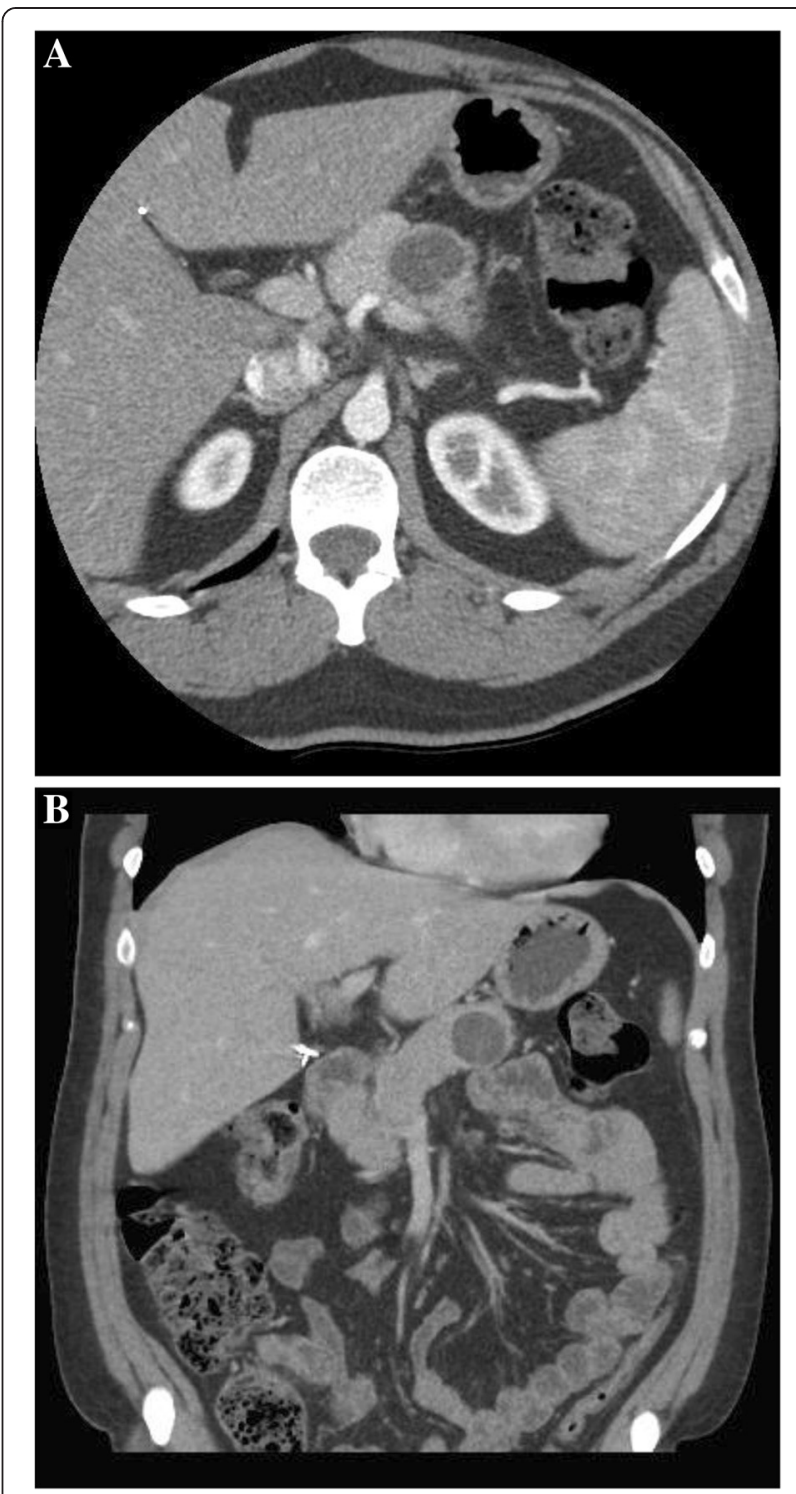

Fig. 1 Abdominal CT with pancreatic protocol showing cystic pancreatic mass. a Arterial phase CT axial slice through the pancreatic body shows a cystic mass within the body region. b Coronal reconstruction portal venous phase shows a cystic mass confined to the pancreatic parenchyma

pancreatic abscess and fistula, which resolved following endoscopic placement of a pancreatic ductal stent. On yearly follow-up, the patient had no evidence of recurrent disease.

\section{Discussion}

Pancreatic MCN is a HRTP with predominance in females [1], characterized by ovarian-like stroma [4]. Figure 5 outlines the possible mechanisms for the pathogenesis of pancreatic MCN in our patient, which is likely multifactorial. Investigators have speculated about the interaction between HRT and the development of hormone-related 


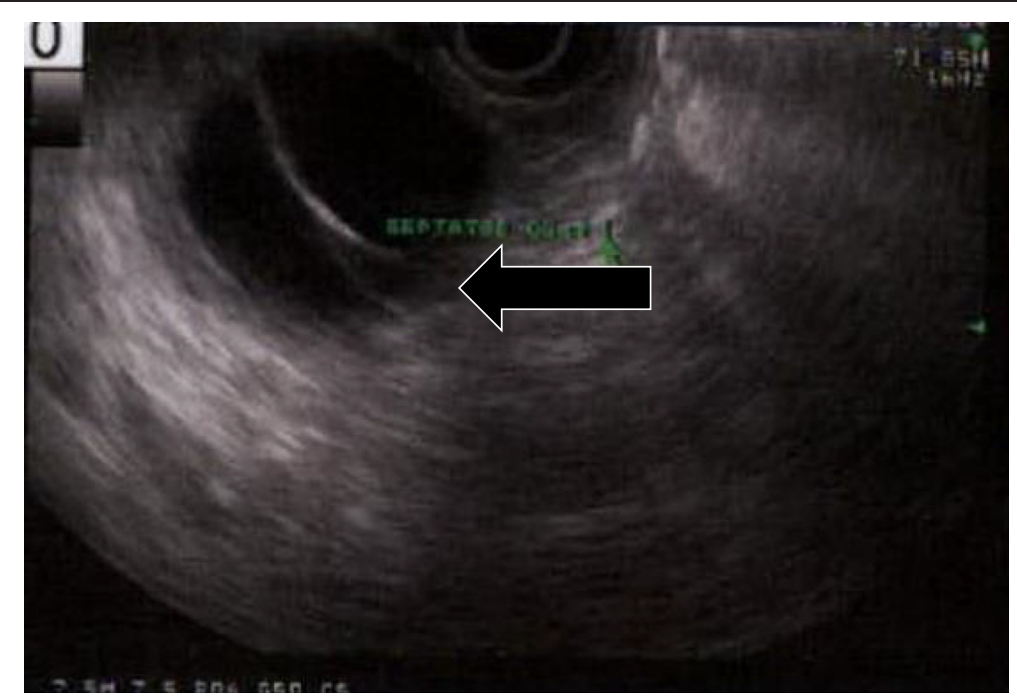

Fig. 2 FNA-EUS showing hypo-echoic pancreatic mass, arrow
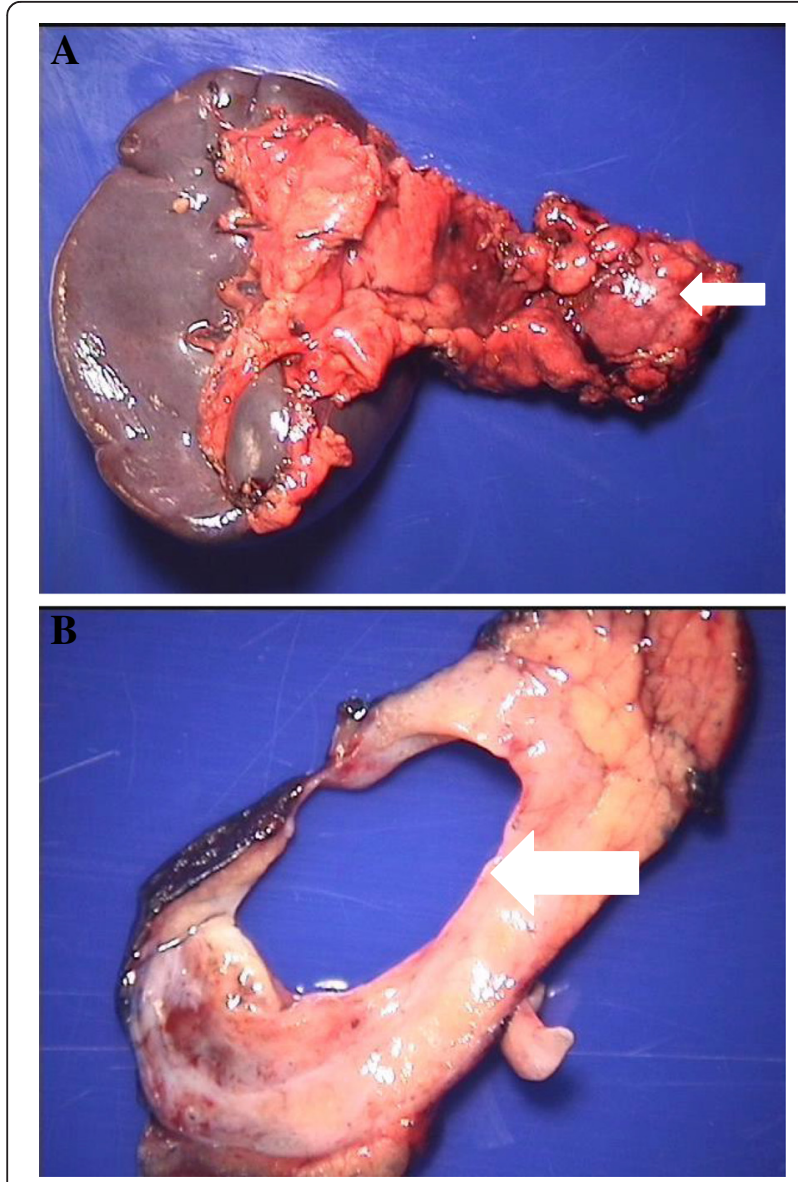

Fig. 3 Gross pathology of the tumor. a A distal portion of pancreas with an attached intact spleen, white arrow delineates pancreatic MCN. b A gross section of pancreatic cyst, white arrow delineates pancreatic cyst wall cancers for many years. Among transgendered females, there are several case reports describing the occurrence of prostate cancer after many decades of estrogen therapy $[14,15]$. The exact role that estrogen plays in the development of pancreatic MCN is not entirely understood [16]. However, the pancreas is known to express estrogen receptors and binding proteins [5]. Normal pancreatic tissue can convert estrone and estrone sulfate into active 17beta-estradiol, and estrogen is believed to be necessary for pancreatic enzyme synthesis [5]. There are contributory reports of MCN development during pregnancy [6-10] and in post-menopausal women on HRT [11].

One explanation for the pathogenesis of pancreatic MCN expressing ovarian-like stroma is that genital gland and dorsal pancreatic buds are adjacent during the course of development such that primary yolk cells may be implanted in the pancreas [16]. This mechanism is plausible in the patient presented here. Another explanation is that immature pancreatic stroma, which expresses sex hormone receptors, responds to female hormones resulting in cystic change and progression [16]. In either case, estrogen is required to stimulate tissue proliferation. The patient discussed here is femaleto-male transgendered on chronic testosterone therapy. It is plausible that exogenous testosterone could have been aromatized to estradiol. This would be particularly true if the testosterone dosing was relatively high. Aromatase and 5-alpha-reductase have both been identified in human pancreatic cancer tissue [17]. The former enzyme converts testosterone to 5-dihydrotestosterone, which is a strong androgen, and the latter enzyme converts testosterone to estradiol as well as delta-4-androstenedione to estrone. Importantly, notable variations in size of our patient's pancreatic $\mathrm{MCN}$ may reflect variation in the 

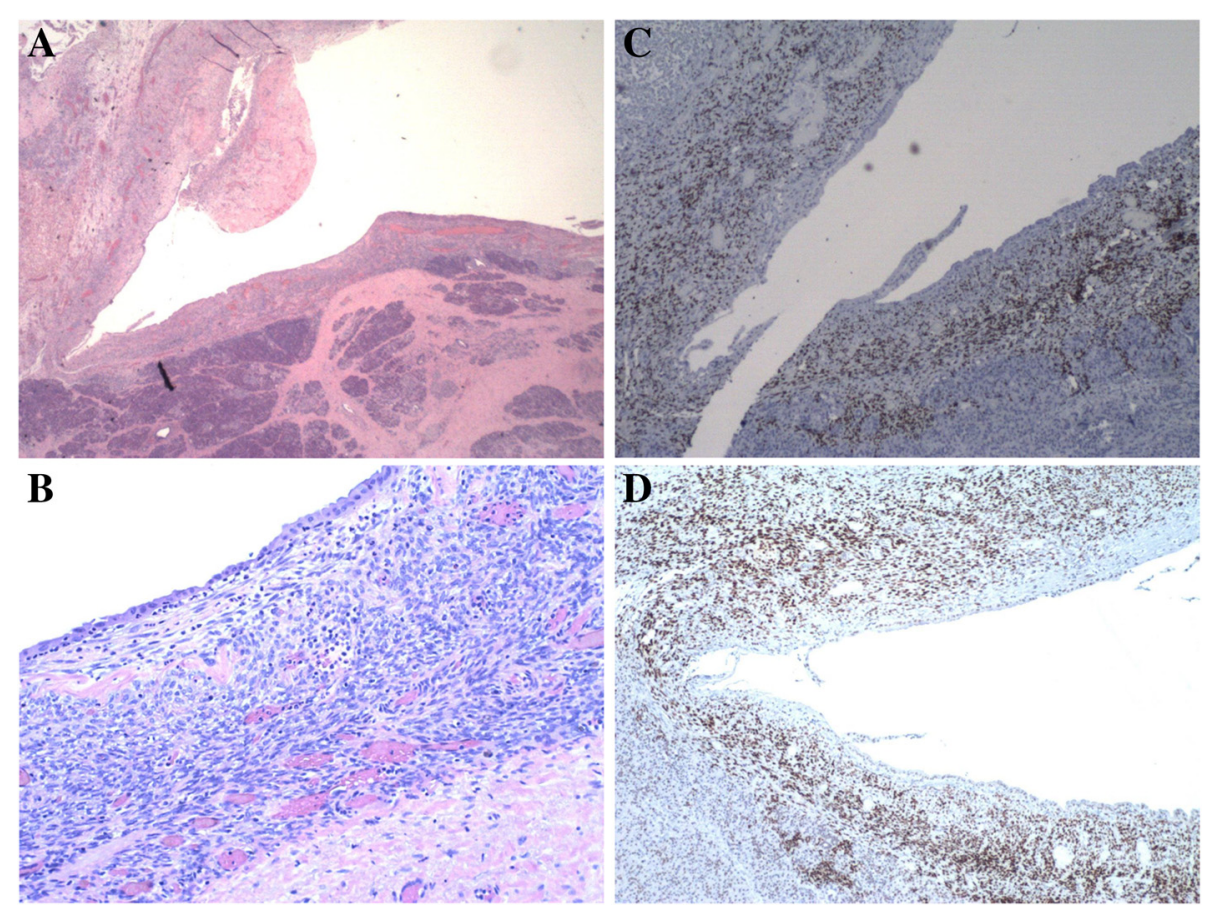

Fig. 4 Microscopic pathology of the tumor. a Pancreatic MCN lined by cuboidal epithelium surrounded by the ovarian-like stroma (H\&E stain; c $\times 1.25)$. $\mathbf{b}$ High-power of pancreatic MCN lined by cuboidal epithelium surrounded by the ovarian-like stroma (H\&E stain; $\mathbf{d} \times 10)$. c Immunohistochemical staining for estrogen receptor within the MCN stroma (4x). d Immunohistochemical staining for progesterone receptor within the MCN stroma (4x)

hormonal environment from estrogen to testosterone, which lends credence to the hypothesis of hormonal influences on MCN development and natural history.

The aforementioned embryological mechanism, however, does not fully account for the albeit rare occurrence of pancreatic $\mathrm{MCN}$ in men [1, 12]. The literature concerning pancreatic MCN in males is limited, and it is possible that the development of $\mathrm{MCN}$ in men may not necessarily follow the same pathogenesis as occurs in women [12]. However, of the reports of men with $\mathrm{MCN}$, most have a history of pancreatitis and/or aberrant hormone levels and sexual phenotype characteristics $[1,12,13]$. Moreover, in a recent study that included five cases of pancreatic MCN along with four cases of pancreatic solid pseudopapillary neoplasm (SPN) in men, hormonal and/or sexual dysfunction was identified in four of the nine patients [13]. In one case report of a young Japanese man who was found to have a pancreatic

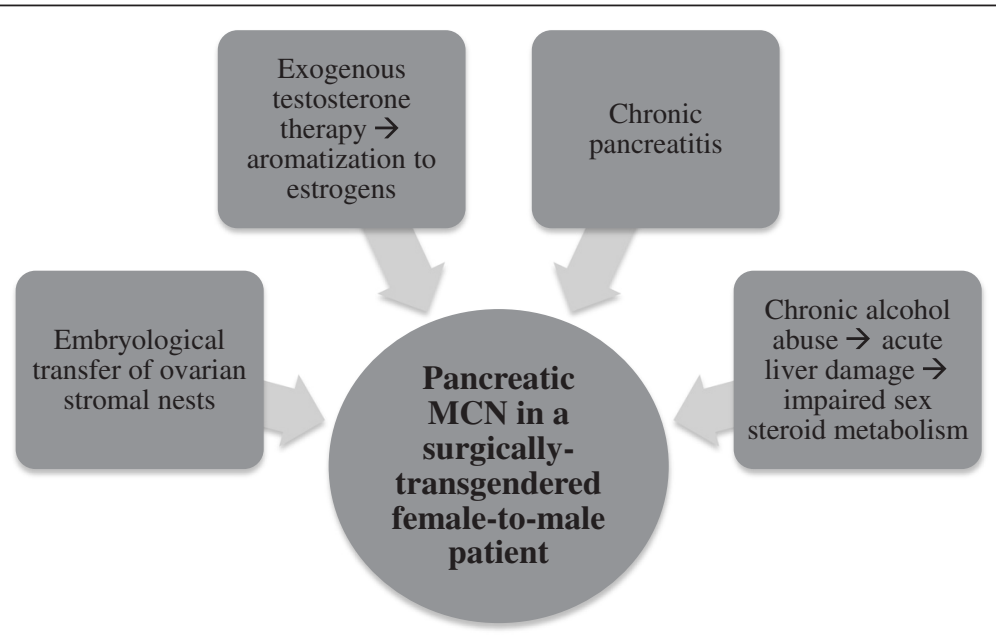

Fig. 5 Proposed multifactorial mechanisms for pathogenesis of pancreatic MCN in a transgender male 
MCN with ovarian-type stroma and antiestrogen and antiprogesterone staining positivity, the authors question whether or not the presence of female hormone receptors is adequate to term the tissue of origin to be ovarian. Pancreatic tissue is also known to express testosterone receptors as well as estrogen receptors [5]. It has been established that there are androgen receptors in pancreatic cancer, and testosterone has been experimentally shown to promote growth of pancreatic adenocarcinoma [5].

Chronic pancreatitis is another proposed risk factor for the development of pancreatic MCN. Wouters et al. published a case report discussing a 43-year-old man who had a 10-year history of moderate to high alcohol intake and chronic pancreatitis that developed a pancreatic MCN [1]. Accordingly, a possible mechanism for the development of pancreatic MCN in chronic alcohol abusers, male or female, is chronic excess alcohol consumption that results in increased estrogen levels either via increased peripheral fat conversion of androgen precursors to estrogen and/or via transient or permanent liver damage resulting in hormonal imbalance secondary to disrupted sex steroid metabolic pathways with elevated estrogen levels [1]. The patient presented in this case had a long history of alcohol abuse.

In terms of management, surgical resection remains the cornerstone for the treatment of high risk pancreatic cystic lesions. Clinicopathologic factors identified as higher risk for malignancy among pancreatic cystic neoplasms includes size greater than $3 \mathrm{~cm}$, presence of septations, a solid component, and related symptomatology $[3,4]$.

\section{Conclusion}

Here, we present the first reported case of a pancreatic MCN in a surgically transgendered female-to-male patient. This patient had a history of alcoholic pancreatitis and was on chronic, long-term testosterone therapy. We provide a case presentation and propose possible mechanisms, based upon our review of the literature that could explain the pathogenesis of his neoplasm. Transgendered patients generally tolerate HRT well. However, this case suggests that pancreatic neoplasms, in part, may be susceptible to HRT. This case also illustrates the importance of being aware of special medical and surgical issues in the transgender population.

\section{Consent}

Informed consent was obtained from the patient for publication of this case report and any accompanying images.

\section{Abbreviations}

CT: computed tomography; EUS: endoscopic ultrasound; HRT: hormone replacement therapy; HRTP: hormone-related pancreatic tumor; IPMN: intraductal papillary mucinous neoplasms; MCN: mucinous cystic neoplasm; SPN: solid pseudopapillary neoplasm.

\section{Competing interests}

The authors declare that they have no competing interests.

\section{Authors' contributions}

DF, MS, EG, BB, JL, RO, MH, DR, and WBB all have made substantial contributions to the conception and design of this study. DF, MS, EG, BB, JL, RO, MH, DR, and WBB all have made substantial contributions to the acquisition of data, as well as analysis and interpretation of data; $\mathrm{DF}, \mathrm{MS}, \mathrm{EG}, \mathrm{BB}, \mathrm{JL}, \mathrm{RO}, \mathrm{MH}, \mathrm{DR}$, and WBB have been involved in drafting the manuscript or revising it critically for important intellectual content; $D F, M S, E G, B B, J L, R O, M H, D R$, and WBB have given final approval of the version to be published and agree to be accountable for all aspects of the work in ensuring that questions related to the accuracy or integrity of any part of the work are appropriately investigated and resolved. All authors read and approved the final manuscript.

\section{Acknowledgements}

We acknowledge and appreciate the willingness of the patient to be featured in this publication. This article did not receive any specific funding. No additional writers participated in the preparation of this article aside from the aforementioned authors.

\section{Author details}

'Division of Surgical Oncology, Department of Surgery, Drexel University College of Medicine, 254 N. 15th St, MS 413, Philadelphia, PA 19102, USA. ${ }^{2}$ Department of Pathology, Drexel University College of Medicine, $254 \mathrm{~N}$. 15th St, MS 435, Philadelphia, PA 19102, USA. ${ }^{3}$ Department of Diagnostic Radiology, Hahnemann University Hospital, 230 N. 15th St, MS 206, Philadelphia, PA 19102, USA. ${ }^{4}$ Department of Medicine, Drexel University College of Medicine, 254 N. 15th St, MS 427, Philadelphia, PA 19102, USA.

Received: 29 April 2015 Accepted: 11 June 2015

Published online: 24 June 2015

\section{References}

1. Wouters K, Ectors N, Van Steenbergen W, Aerts R, Driessen A, Van Hoe L, et al. A pancreatic mucinous cystadenoma in a man with mesenchymal stroma, expressing oestrogen and progesterone receptors. Virchows Arch. 1998:432(2):187-9.

2. Goh BKP, Tan Y-M, Chung Y-FA, Chow PKH, Cheow P-C, Wong W-K, et al. A review of mucinous cystic neoplasms of the pancreas defined by ovariantype stroma: clinicopathological features of 344 patients. World J Surg. 2006;30:2236-45.

3. Allen PJ, D'Angelica M, Gonen M, Jaques DP, Coit DG, Jarnagin WR, et al. A selective approach to the resection of cystic lesions of the pancreas. Ann Surg. 2006;244:572-82.

4. Allen PJ, Jaques DP, D'Angelica M, Bowne WB, Conlon KC, Brennan MF. Cystic lesions of the pancreas: selection criteria for operative and nonoperative management in 209 patients. J Gastrointest Surg. 2003;7:970-7.

5. Andrén-Sandberg A, Hoem D, Bäckman PL. Other risk factors for pancreatic cancer: hormonal aspects. Ann Oncol. 1999;10 Suppl 4:131-5.

6. Herring AA, Graubard MB, Gan SI, Schwaitzberg SD. Mucinous cystadenocarcinoma of the pancreas during pregnancy. Pancreas. 2007;34:470-3.

7. Ikuta S. Large mucinous cystic neoplasm of the pancreas associated with pregnancy. World J Gastroenterol. 2008;14:7252.

8. Ishikawa K, Hirashita T, Kinoshita H, Kitano M, Matsuo S, Matsumata T, et al. Large mucinous cystadenoma of the pancreas during pregnancy: report of a case. Surg Today. 2007;37:1013-7.

9. Kato M, Kubota K, Kita J, Shimoda M, Rokkaku K, Inaba N, et al. Huge mucinous cystadenoma of the pancreas developing during pregnancy: a case report. Pancreas. 2005;30:186-8.

10. Smithers BM, Welch C, Goodall P. Cystadenocarcinoma of the pancreas presenting in pregnancy. Br J Surg. 1986;73:591.

11. Tanaka S, Kawamura T, Nakamura N, Teramoto K, Arii S. Mucinous cystadenocarcinoma of the pancreas developing during hormone replacement therapy. Dig Dis Sci. 2007;52:1326-8.

12. Suzuki M, Fujita N, Onodera H, Kayaba Y, Suzuki S, Kagaya H, et al. Mucinous cystic neoplasm in a young male patient. J Gastroenterol. 2005;40:1070-4.

13. Regi P, Salvia R, Cena C, Girelli R, Frigerio I, Bassi C. Cystic "feminine" pancreatic neoplasms in men. Do any clinical alterations correlate with these uncommon entities? Int J Surg. 2013;11:157-60. 
14. Turo R, Jallad S, Prescott S, Cross WR. Metastatic prostate cancer in transsexual diagnosed after three decades of estrogen therapy. Can Urol Assoc J. 2013;7(7-8):E544-6.

15. Miksad RA, Bubley G, Church P, Sanda M, Rofsky N, Kaplan I, et al. Prostate cancer in a transgender woman 41 years after initiation of feminization. JAMA. 2006;296:2316-7.

16. Zamboni G, Scarpa A, Bogina G, lacono C, Bassi C, Talamini G, et al. Mucinous cystic tumors of the pancreas: clinicopathological features, prognosis, and relationship to other mucinous cystic tumors. Am J Surg Pathol. 1999;23:410-22.

17. labal MJ, Greenway B, Wilkinson ML, Johnson PJ, Williams R. Sex-steroid enzymes, aromatase and 5 alpha-reductase in the pancreas: a comparison of normal adult, foetal and malignant tissue. Clin Sci (Lond). 1983;65:71-5.

\section{Submit your next manuscript to BioMed Central and take full advantage of:}

- Convenient online submission

- Thorough peer review

- No space constraints or color figure charges

- Immediate publication on acceptance

- Inclusion in PubMed, CAS, Scopus and Google Scholar

- Research which is freely available for redistribution 